\title{
Assessment of Injuries of Tempo ro-Mandibular Joint by MRI
}

\author{
SAMEH M. ABDELWAHAB, M.D.; MENNAT ALLAH H. SHALABY, M.D. and \\ MAGED A. ABDULLAH BAHATHEQ, M.Sc.
}

The Department of Radiodiagnosis, Faculty of Medicine, Ain Shams University

\begin{abstract}
Background: The use of magnetic resonance imaging (MRI) in the assessment of temporomandibular joint (TMJ) injuries has been a mainstay. Since it can non-invasively showing major regional anatomic structures and revealing the presence of joint effusion and bone marrow signal-intensity abnormalities.
\end{abstract}

Aim of Study: To elucidate the role of MRI in the diagnosis of TMJ injuries and for which extent MRI is able to detect its abnormalities.

Patients and Methods: A total of 43 patients with TMJ injuries were included in the study. A 1.5 tesla MRI machine is used to assess the TMJs. The position of the disc, its configuration, the presence or absence of joint effusion, osteoarthritis, and the morphology of the mandibular condyle were all evaluated.

Results: The clinical symptoms of the patients there were higher of symptoms in sensation of pain $(86 \%)$; followed by limited mouth opening (44.2\%). The patients anterior disc dislocation with reduction was higher value was found in patients' injuries of temporomandibular joint 17 patients $(39.5 \%)$ with $p$-value $<0.001$ highly significant, followed by 8 patients (18.6\%) in anterior disc dislocation with no reduction when opening the mouth, with $p$-value 0.003 significant. The patient's degenerative changes of the disc with signal intensity was higher value was found in patients' injuries of temporomandibular joint 31 patients $(72.1 \%)$ with $p$-value $<0.001$ highly significant, followed by 16 patients $(37.2 \%)$ in degenerative changes of the disc with disc shape, with p-value 0.001 significant. The patients other finding with osteoarthritic change was higher value was found in patients' injuries of temporomandibular joint 13 patients $(30.2 \%)$ with $p$-value $<0.001$ highly significant, followed by 7 patients $(16.3 \%)$ in hypermobility, with $p$-value 0.006 significant, all these results indicate a strong relationship in the assessment by MRI.

Conclusion: Disc displacement or internal disc derangement is the most prevalent disorder. MRI is the proper diagnostic modality for TMJ disorders due to non-invasive technique, excellent soft tissue contrast and multi-planner capabilities.

Correspondence to: Dr. Sameh M. Abdelwahab, The Department of Radiodiagnosis, Faculty of Medicine, Ain Shams University
Key Words: Temporomandibular joint disorders - Magnetic resonance imaging - Disc displacement - Internal derangement - Joint effusion.

\section{Introduction}

TMJ is the most frequently widely used in the body, for example in chewing and talking. Injuries of TMJ is a common condition affecting around $28 \%$ of population. The incidence of TMJ injuries are highly reported on female and the ratio may reach up to 8:1 compared to male. Moreover, the symptoms may present between 20-50 years of age in most patients [1]. TMJ injuries are musculoskeletal degeneration with association of functional and morphological conditions either caused by abnormalities of TMJ components or related to structures such as ligaments, muscles, periodontal tissue and teeth [2]. Internal derangement (ID) is the most common TMJ injuries. It may affect around $70 \%$ of patients. It characterized by dislocated disc or pathology. Other causes of TMJ injuries including, osteoarthrosis, inflammatory condition such as juvenile inflammatory arthritis and rheumatoid arthritis, trauma, and rarely development abnormalities, and neoplasms [3]. Factors such as trauma, occlusal abnormalities, sleep abnormality, systemic predisposing factors, parafunctional habits and psychosocial deleterious changes, considered as risk factors of TMJ disorders $[4,5]$. Signs of TMJ injuries may be present as muscular or joints pain, limitation of mouth activities, or clicking sounds usually painless. The patients suffer from pain or clicking sound during mouth opening or closing movement, limitation of jaw opening and TMJ locking, migraine, headache, or pain around the era. Sometimes especially in early stages of TMJ injuries, the patients may be asymptomatic [3]. MRI is the modality of choice for diagnosis of TMJ injuries because of its ability for better visualization of small structure of TMJ component as retrodiscal layer and lateral pterygoid 
attachment, also MRI can evaluate the morphological state of TMJ and analysis of dynamic process (it is pseudo dynamic MR imaging obtained from serial multiple static images). Other advantages of MRI include, detection of soft tissue changes, necrosis, joint effusion, and bone marrow abnormalities, non-invasive procedure, no risk of radiation exposure, diagnostic accuracy, high sensitivity and specificity. MRI is the most advanced imaging modality of choice for diagnosis of TMJ injuries or disorder and the gold standard in disc displacement diagnosis [6].

\section{Patients and Methods}

In this retrospective study, which included 43 consecutive patients, who were referred from Department of Diagnostic Radiology at Ain Shames University. The mean age of the patients was 26.88 \pm 10.92 years (range $15-70$ years). There were 33 $(76.7 \%)$ female and $10(23.3 \%)$ male of sex, they were suffering from clinical manifestations of temporo-mandibular joint injuries. This study was performed at the period from March 2020 to April 2021. All patients with the following clinical manifestations of TMJ injuries were included in this study: Pain in or around the TMJ during mandibular movement, limited jaw opening ability, TMJ locking, TMJ clicking sounds during mouth opening and/or closing movement. Patients with the following manifestations were excluded from the study: Patient with cerebral aneurysm clip, patient with cardiac pacemaker, patient who were claustrophobic and uncooperative, metallic prosthesis heart valves, ferromagnetic foreign bodies in critical location like eye (intra-ocular foreign bodies) and cochlea (cochlear implants), patient with obvious skeletal jaw deformity, patient undergoing/history of orthodontic treatment, patient with history of traumatic extraction. The main tool for diagnostic assessment was the conventional MR study of the TMJ in both opened and closed mouth positions.

\section{Conventional MR study:}

Routine bilateral TMJ examination on 1.5 Tesla machine (Philips). The patient lies supine, head first, both arms are adducted, with applied of special TMJ dual coil. In both of closed and maximum open mouth position, pulse sequences were obtained from all patients on corrected (oblique) sagittal T1WI, proton density (PD) and T2WI with corrected (oblique) coronal cuts in T 1 and PD weighted image. All pulse sequence has the same parameters which is as follows:

- Supine position with head first.

- Coil type: Dual.
- Image mode: 2D.

- Pulse sequence: Spin echo.

- Slice thickness: $3 \mathrm{~mm}$ with no spatial gab.

- Number of slices: 18.

- Matrix: 256 x 128.

- Field of view: 10-12.

- Imaging time: $3.14 \mathrm{~min}$.

The MRI of TMJ begins with axial localizer including the whole skull.

TMJ localizer:

- Axial plane of spin echo pulse sequence with 10 slices number.

- Slice thickness/space: $5 / 2 \mathrm{~mm}$.

- TR/TE: $300 \mathrm{~ms} / 12 \mathrm{~ms}$.

- Imaging time: $28 \mathrm{~s}$.

Specific parameters for sagittal and coronal oblique T1 WI:

- Repetition time: 400-50msec.

- Echo time: 10-20msec.

Specific parameters for sagittal and coronal oblique PDWI:

- Repetition time: $2000 \mathrm{msec}$.

- Echo time: 10-14msec.

Specific parameters for sagittal oblique T2WI:

- Repetition time: 2600msec.

- Echo time: $120 \mathrm{msec}$.

Dynamic images: Formed as in during progressive opening and closing the mouth a rapid question of static images was obtained by use of a single shot fast echo (SSFSE) T2WI. Eight sequence are obtained and performed in straight sagittal orientation along anticipated path of condylar motion. Then, inserting the images sequentially as a cine loop.

\section{Statistical analysis:}

Recorded data were analyzed using the statistical package for social sciences, version 20.0 (SPSS Inc., Chicago, Illinois, USA). Quantitative data were expressed as mean \pm standard deviation (SD). Qualitative data were expressed as frequency and percentage.

The following tests were done:

- Chi-square $\left(\mathrm{x}^{2}\right)$ test of significance was used in order to compare proportions between qualitative parameters. 
- The confidence interval was set to $95 \%$ and the margin of error accepted was set to $5 \%$. So, the $p$-value was considered significant as the following:

- Probability ( $p$-value).

- $p$-value $<0.05$ was considered significant.

- $p$-value $<0.001$ was considered as highly significant.

- $p$-value >0.05 was considered insignificant.

\section{Results}

The results of the present study are demonstrated in the following Tables (1-3) and Figs. (1,2). We aimed by our study to elucidate the role of MRI in the diagnosis of TMJ injuries and for which extent MRI is able to detect its abnormalities, we applied our study on 43 patients with the same inclusion and exclusion criteria. The mean age of the patients was $26.88 \pm 10.92$ years (range 15-70 years). There were $33(76.7 \%)$ female and 10 $(23.3 \%)$ male of sex.

The clinical symptoms of the patients there were higher of symptoms $37(86 \%)$ in sensation of pain; followed by 19 (44.2\%) limited mouth opening; and 14 (32.6\%) joint noise, while the lowest symptoms were found in deflection / deviation $1(2.3 \%)$.

The patients anterior disc dislocation with reduction was higher value was found in patients' injuries of temporomandibular joint 17 patients $(39.5 \%)$ with $p$-value $<0.001$ highly significant, followed by 8 patients $(18.6 \%)$ in anterior disc dislocation with no reduction when opening the mouth, with $p$-value 0.003 significant, this indicates a strong relationship in the assessment by MRI.

The patient's degenerative changes of the disc with signal intensity was higher value was found in patients' injuries of temporomandibular joint 31 patients $(72.1 \%)$ with $p$-value $<0.001$ highly significant, followed by 16 patients (37.2\%) in degenerative changes of the disc with disc shape, with $p$-value 0.001 significant, this indicates a strong relationship in the assessment by MRI.

The patients other finding with osteoarthritic change was higher value was found in patients' injuries of temporomandibular joint 13 patients $(30.2 \%)$ with $p$-value $<0.001$ highly significant, followed by 7 patients $(16.3 \%)$ in hyper mobility, with $p$-value 0.006 significant, this indicates a strong relationship in the assessment by MRI.

While the lowest value of patients at other finding with sclerosis 3 (7\%), joint effusion 2
(4.7\%), with $p$-value $>0.05$ non-significant. As for the Congenital anomalies and Mass or tumor like lesions, there are no diagnosed cases through MRI.

Table (1): Distribution of patients' injuries of temporomandibular joint according to their finding by MRI regarding anterior disc dislocation "With reduction and with no reduction when opening the mouth" $(n=43)$.

\begin{tabular}{lcccc}
\hline Anterior disc dislocation & No. & $\%$ & $\mathrm{x}^{2}$ & $p$-value \\
\hline With reduction: & & & & \\
$\quad$ No & 26 & 60.5 & 20.919 & $<0.001^{* *}$ \\
$\quad$ Yes & 17 & 39.5 & & \\
$\begin{array}{l}\text { With no reduction when } \\
\text { opening the mouth: }\end{array}$ & & & & \\
$\quad$ No & 35 & 81.4 & 8.716 & $0.003^{*}$ \\
$\quad$ Yes & 8 & 18.6 & & \\
\hline
\end{tabular}

Using: Chi-square test. ${ }^{*} p$-value $<0.05 \mathrm{~S} . * * p$-value $<0.001 \mathrm{HS}$.

Table (2): Distribution of patients' injuries of temporomandibular joint according to their finding by MRI regarding degenerative changes of the disc "With reduction and with no reduction when opening the mouth" $(=43)$.

\begin{tabular}{lcccc}
\hline $\begin{array}{l}\text { Degenerative changes of } \\
\text { the disc }\end{array}$ & No. & $\%$ & $\mathrm{x}^{2}$ & $p$-value \\
\hline Disc shape changes: & & & & \\
$\quad$ No & 27 & 62.8 & 19.423 & $<0.001^{* *}$ \\
$\quad$ Yes & 16 & 37.2 & & \\
$\quad \begin{array}{lllll}\text { Signal intensity changes: } \\
\quad \text { No }\end{array}$ & 12 & 27.9 & 47.916 & $<0.001^{* *}$ \\
$\quad$ Yes & 31 & 72.1 & & \\
\hline
\end{tabular}

Using: Chi-square test. $* * p$-value $<0.001 \mathrm{HS}$.

Table (3): Distribution of patients' injuries of temporomandibular joint according to their finding by MRI regarding other finding "Osteoarthritic change, joint effusion, hyper mobility, sclerosis, congenital anomalies and mass or tumor like lesions" ( $\mathrm{n}=43)$.

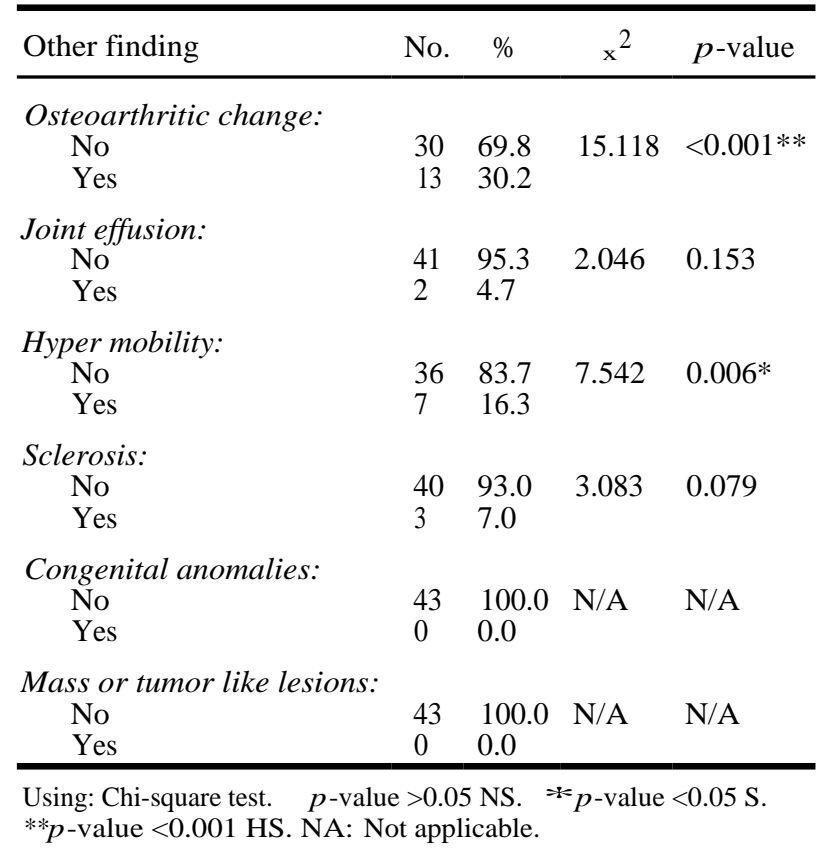



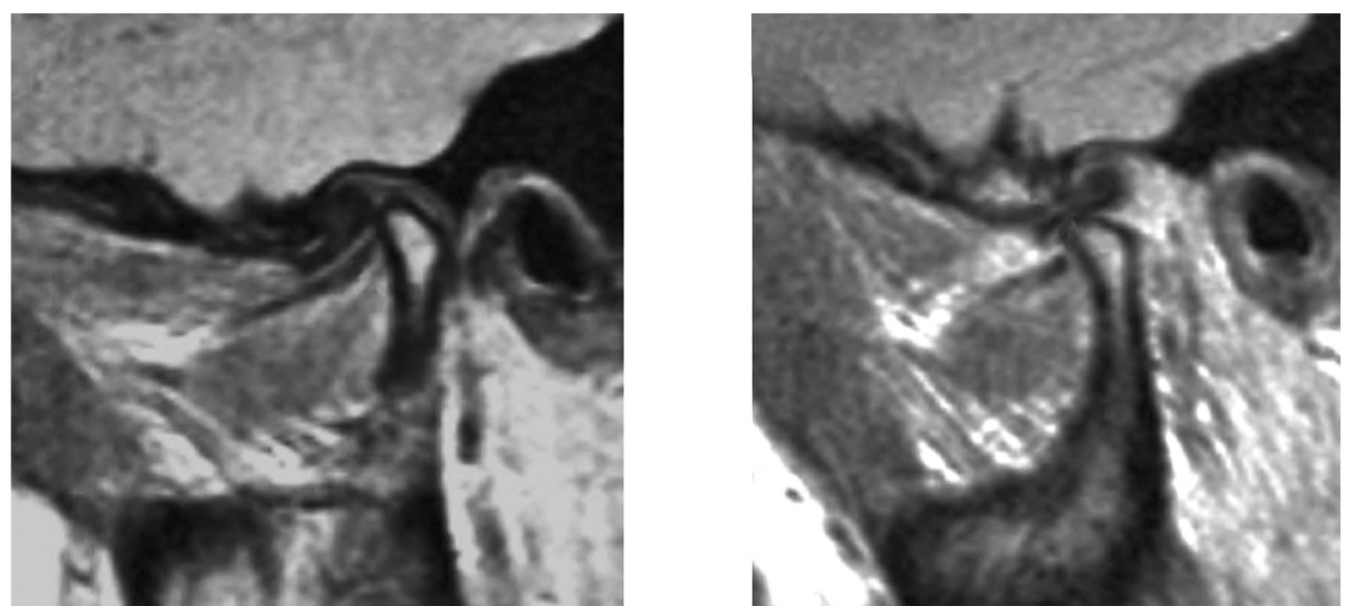

Fig. (1): Pie chart distribution of patients' injuries of temporomandibular joint according to their sex.
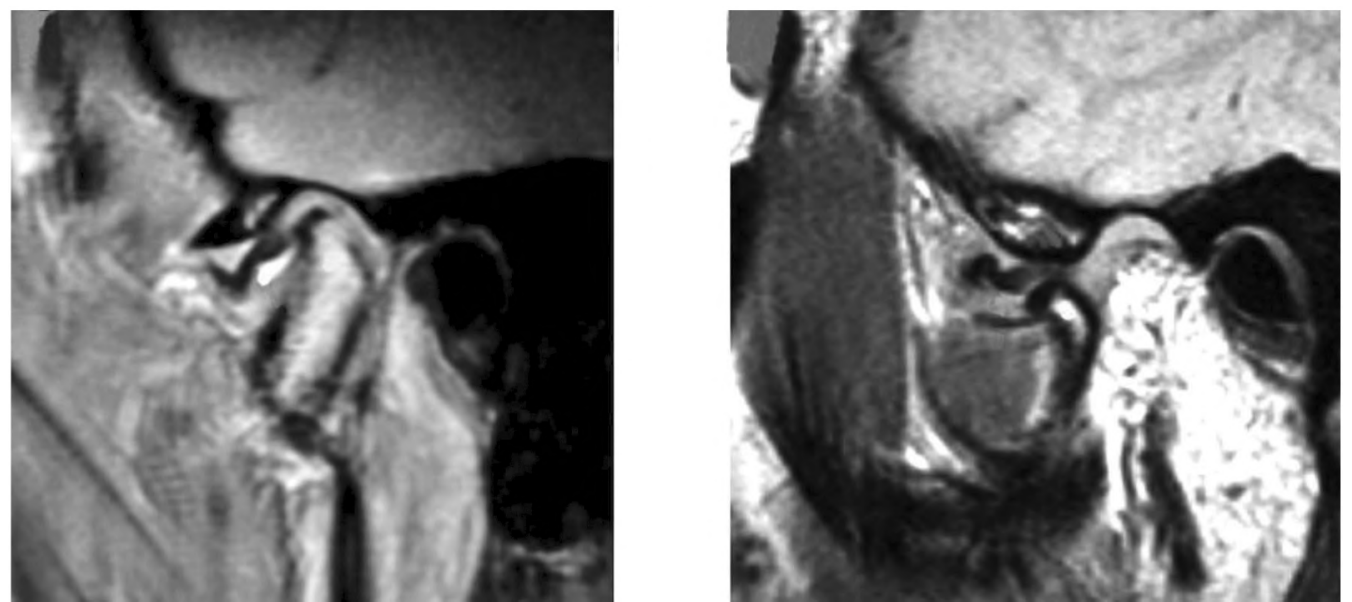

Fig. (2): Bar chart distribution of patients' injuries of temporomandibular joint according to their clinical symptoms.

\section{Discussion}

Temporomandibular joint (TMJ) pain and dysfunction are important clinical problems which are commonly seen, affect up to $28 \%$ of population [1].

Major components of the TMJ include the mandibular condyle, the articular disc, the glenoid fossa, and the articular eminence of the temporal bone. Unlike most joints, the articulating surfaces are fibrous and not cartilaginous. The fibro cartilaginous articular disc is biconcave, dividing the joint space into superior and inferior compartments [7].

Internal derangement is the most common TMJ injuries, it affects around $70 \%$ of patients. Other causes of TMJ injuries including osteoarthrosis, inflammatory conditions, trauma, and parafunctional habits, rarely anatomical variation and mass or tumor like lesions [4,5].
The accuracy of clinical examination for diagnosing the exact status of TMJ internal derangements has been investigated in several previous studies [8], which reported that the overall accuracy of the clinical examination was $43 \%$ to $95 \%$. False positive diagnoses are related to over classification of the disease, which might lead to over-treatment, while false negative diagnoses are related to underclassification and consequently under-treatment.

MRI is the modality of choice for diagnosis of TMJ injuries because of its ability for better visualization of small structure of TMJ component, evaluation of the morphological state of TMJ and analysis of dynamic process, excellent soft tissue contrast and no radiation exposure [6].

The current work included 43 patients with symptoms and signs of TMJ injuries as follows: Joint pain, noise sensation, limited mouth opening or jaw deviation. 
In the present work, sex showed statistically significant differences between the studied groups. Out of total 43 patients studied, 33 were females and 10 were males. The ratio of female to male in this group of patients with temporomandibular joint disorders was 3.3:1. Higher female ration in this study agrees with that of Amin et al., who found increased ratio of female to male patients with temporomandibular joint dysfunctions $(2.5: 1)$ [8]. Other study by Okeson reported with comparable results [9].

Another study done by Dalkiz et al., showing that pain and dysfunction TMJ injuries seem to affect women more than men with clinical reports has emphasized the high ratio $(8.67: 1)$ of female to male patients for TMJ injuries [10].

In current study, the most common clinical sign and symptom of the examined patient's group was pain in preauricular region (37 out of 43 patients) representing $86 \%$ with regard to the patients who suffer TMJ pain, and numerous studies based on examination of the MR findings have been carried out. The main findings that are responsible for the etiology are disk malposition, effusion and osteoarthrosis [11]. This agrees with those of Okeson who reported that disk displacement of TMJ is the important cause of facial and TMJ pain [9] .

On the contrary, Farina et al., in his study showing that MR signal changes in retrodiscal tissue are highly correlated with TMJ pain [12]

In the present study, joint noise was found in 14 out of 43 patients (33\%), thus indicating that TMJ clicking might occur as a consequence of frictional incompatibility between disk and the eminence, when the posterior band of the disk moves anteriorly or posteriorly beyond the apex of the articular eminence. Other causes may be deviation in condylar form (remodeling), adhesion or muscular incardination. Our explanation goes with the study done by Imanimoghaddam et al., who concluded that strong correlation exists between disk displacement on MRI and TMJ click [13].

In our study bony osteoarthritic changes were diagnosed in 13 patients out of 43 representing $30 \%$. This agrees with those of Emshoff et al., and they found a significant relationship between TMJ internal derangement and osteoarthritis and bone marrow edema as well as a significant increase in risk of pain [14].

In the present study the incidence of disk displacement was noted in 17 patients representing
$39.5 \%$ compared to $86 \%$ in the study done by Emshoff and coworkers [14].

In our study, $60 \%$ of studies cases showed no disk displacement. In study done by Samara et al., revealed that $30 \%$ of the studied cases shows no abnormalities on MRI examination [15] .

In this study, the patients anterior disc dislocation with reduction was higher value was found in patients' injuries of temporomandibular joint 17 patients $(39.5 \%)$ with $p$-value $<0.001$ highly significant, followed by 8 patients $(18.6 \%)$ in anterior disc dislocation with no reduction when opening the mouth, with $p$-value 0.003 significant, this indicates a strong relationship in the assessment by MRI. Compared to study done by Amin et al., on 28 joints examined by MRI, 8 joints showed disk displacement with reduction $(28.7 \%)$, while 16 joints showed disk displacement without reduction $(57.1 \%)$ [8]

In present study, all disc displacement cases were anteriorly displaced and no sideway (lateral or medial) or posterior displacement has been discovered, this agrees with a study done by Milano et al., who found that sideway disk displacement is rare $(1.5 \%)$ in their study [16].

In our study, the patient's degenerative changes of the disc with signal intensity was higher value was found in patients' injuries of temporomandibular joint 31 patients $(72.1 \%)$ with $p$-value $<0.001$ highly significant, followed by 16 patients $(37.2 \%)$ in degenerative changes of the disc with disc shape, with $p$-value 0.003 significant, this indicates a strong relationship in the assessment by MRI.

In a study done by Yilmaz \& Toller, they were evaluating 133 temporomandibular joints by MRI, they found that the articular disk is deformed in $52.38 \%$ of the joints with reduced anteriorly displaced disk whereas $85.71 \%$ of the non-reducible disks were deformed [17].

\section{Conclusion:}

Disc displacement or internal disc derangement is the most prevalent disorder. MRI is the proper diagnostic modality for TMJ injuries due to noninvasive technique, excellent soft tissue contrast and multi-planner capabilities.

\section{References}

1- MARTINS-JÚNIOR R.L., et al.: Temporomandibular disorders: A report of 124 patients. J. Contemp Dent Pract, 11 (5): p. 071-8, 2010.

2- MURPHY M.K., et al.: Temporomandibular disorders: A review of etiology, clinical management, and tissue engi- 
neering strategies. Int. J. Oral Maxillofac. Implants, 28 (6): p. e393-414, 2013.

3- PETS CAVAGE-THOMAS J.M. and E.A. WALKER: Unlocking the jaw: Advanced imaging of the temporomandibular joint. AJR Am. J. Roentgenol., 203 (5): p. 1047-58, 2014.

4- CARRARA S.V. and C.P., BARBOSA J.S.: Statement of the 1 st Consensus onTemporomandibular Disorders and Orofacial Pain, Dent Press J. Orthod., 15: 114-120, 2010.

5- HUNTER A. and S. KALATHINGAL: Diagnostic imaging for temporomandibular disorders and orofacial pain. Dent Clin. North Am., 57 (3): p. 405-18, 2013.

6- TOMAS X., et al.: MR imaging of temporomandibular joint dysfunction: A pictorial review. Radiographics, 26 (3): p. 765-81, 2006.

7- WAND E.Y. and K.A. FLEISHER: MRI of temporomandibular joint disorders. Applied radiology, 37 (9): p. 17, 2008.

8- AMIN M.F., A.M. HASSAN and K.I. BARAKAT: The accuracy of dynamic Magnetic Resonance Imaging in evaluation of internal derangement of the temporomandibular joint; comparison with arthroscopic findings. The Egyptian Journal of Radiology and Nuclear Medicine, 43 (3): p. 429-436, 2012.

9- JP O.: Management of Temporomandibular Disorders and Occlusion, 2003.

10- DALKIZ M., E. PAKDEMIRLI and B. BEYDEMIR: Evaluation of temporomandibular joint dysfunction by magnetic resonanance imaging. Turkish Journal of Medical Sciences, 31 (4): p. 337-343, 2001.
11- CHIBA M., M. KUMAGAI and S. ECHIGO: Association between high signal intensity in the posterior disc attachment seen on T 2 weighted fat-suppressed images and temporomandibular joint pain. Dentomaxillofacial Radiology, 36 (4): p. 187-191, 2007.

12- FARINA D., et al.: TMJ disorders and pain: assessment by contrast-enhanced MRI. European journal of radiology, 70 (1): p. 25-30, 2009.

13-IMANIMOGHADDAM M., A.S. MADANI and E. MAHMOUDI HASHEMI: MRI findings in patients with TMJ click. Journal of Dental Materials and Techniques, 3 (1): p. 28-36, 2013.

14-EMSHOFF R., et al.: Magnetic resonance imaging findings of internal derangement, osteoarthrosis, effusion, and bone marrow edema before and after performance of arthrocentesis and hydraulic distension of the temporomandibular joint. Oral Surgery, Oral Medicine, Oral Pathology, Oral Radiology, and Endodontology, 101 (6): p. 784-790, 2006

15- SAMARA O., et al.: Correlation between clinical and magnetic resonance imaging (MRI) findings in temporomandibular disorders. Journal of Clinical Medicine and Research, 4 (9): p. 109-113, 2012.

16- MILANO V., et al.: Magnetic resonance imaging of temporomandibular disorders: Classification, prevalence and interpretation of disc displacement and deformation. Dentomaxillofacial Radiology, 29 (6): p. 352-361, 2000.

17- TASKAYA-Y1LMAZ, N. and M. ÖGÜTCEN-TOLLER Magnetic resonance imaging evaluation of temporomandibular joint disc deformities in relation to type of disc displacement. Journal of oral and maxillofacial surgery, 59 (8): p. 860-865, 2001.
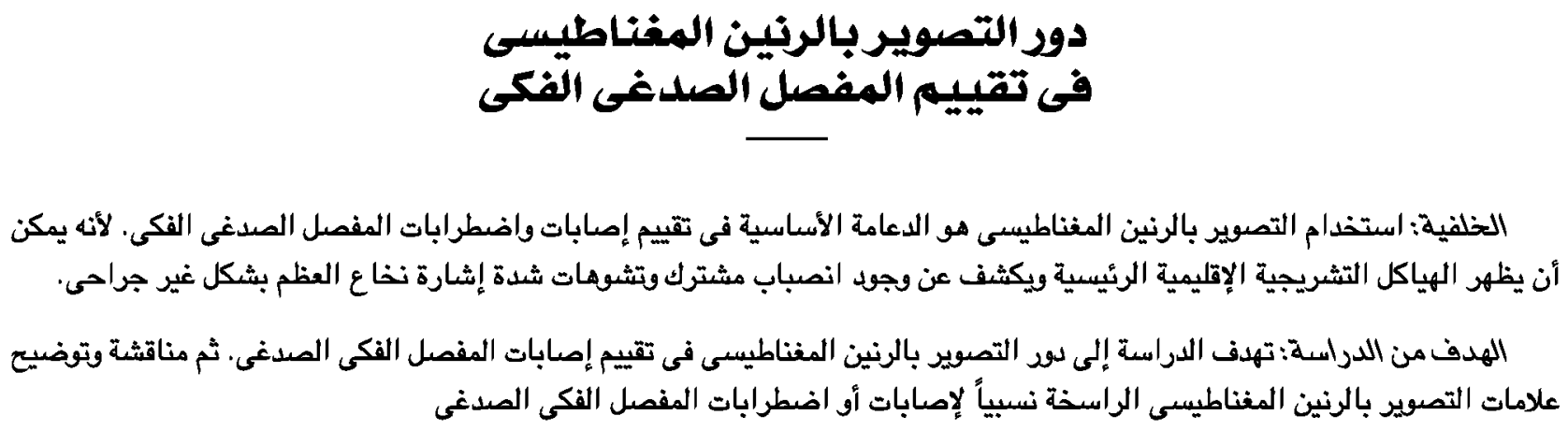

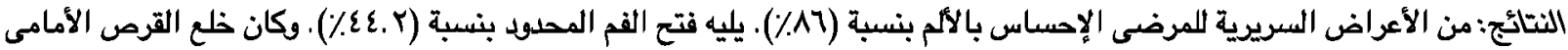

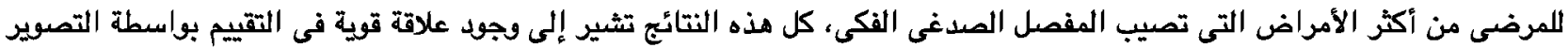
بالرنين المغناطيسى.

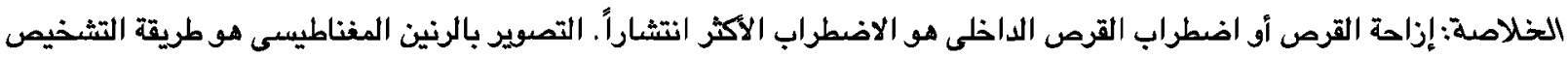

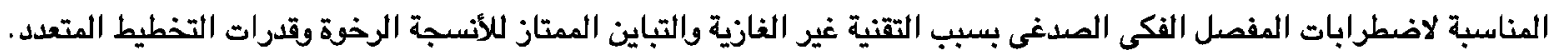

